\title{
Study on Transient Performance Testing Method of Electronic Current Transformer in Smart Substation
}

\author{
WANG Xiao-ming, ZHOU Wei,ZHOU Ke,LI Ming-po,WANG Bin
}

\author{
Electric Power Research Institute Of GUANGXI Power Grid Co., Ltd, Nanning, China \\ Email: wang_xm.sy@gx.csg.cn
}

Keywords: Electronic current transformer,Transient performance,Smart substation,RTDS

\begin{abstract}
Transient performance is an important parameter to measure the performance of electronic current transformer in smart substation, the transient performance of electronic current transformer with analog integrator and digital integrator is analyzed theoretical and simulated, Simulation results show that the transient performance is greatly degraded when integrator parameter or integral algorithm is improperly used. Therefore, it is necessary to test the transient performance. In view of the transient testing limitations of traditional dynamic model test system , a test method based on the technology of RTDS simulation is proposed and the real-time simulation of the transient performance is given, using the method to test the electronic current transformer transient $\mathrm{C}$, the test results show that the method can provide effective reflected in the transient response of the electronic current transformer.
\end{abstract}

\section{Introduction}

As the source of the digital and network transmission of the secondary equipment in the smart substation, the accuracy and reliability of the electronic transformer is closely related to the safety and reliability of the power system. With the continuous development of smart grid construction, the application of electronic transformer is also increasing, at present there are a considerable number of electronic transformers officially hanging network operation(ZHAO Yong\&KONG Shengli,2014 ). Compared to the traditional electromagnetic transformer, the electronic transformer to solve the defects of the insulation and magnetic saturation and the transient response on principle,the secondary side of electronic transformer will output digital signal directly with other intelligent device interface and it will provide the possibility of the secondary equipment networking. But in the application process, there are some problems to be solved, such as: electronic transformer collector under adverse environment interference,stability,reliability, transient performance verification (LI Wei\&YIN Xianggen,2008 and ZHANG Kewei\&WANG Ning,2004 ). Therefore, it is particularly important to research the various testing methods, the electronic transformer test is mainly for its steady performance test, including the ratio error and phase difference, polarity, to study the transient characteristics and test methods, this paper puts forward a method of electronic current transformer transient test based on the technology of RTDS simulation. The method validates the dynamic response of electronic current transformer by state a simulation system of power grid, it provides technical support for the safe and stable operation.

\section{The principle of electronic current transformer}

Currently, the electronic current transformer based on Rogowski coil is the most widely used in the field, which is mainly composed of Rogowski coil as the sensing head, and supplemented by the acquisition module, power module and transmission system. 
The Rogowski coil is air core coil with a special structure where the measuring line winds round the non-magnetic skeleton uniform, the Rogowski coil connected to the output of acquisition module constitutes the electronic current transformer, its structure is shown in Figure 1.

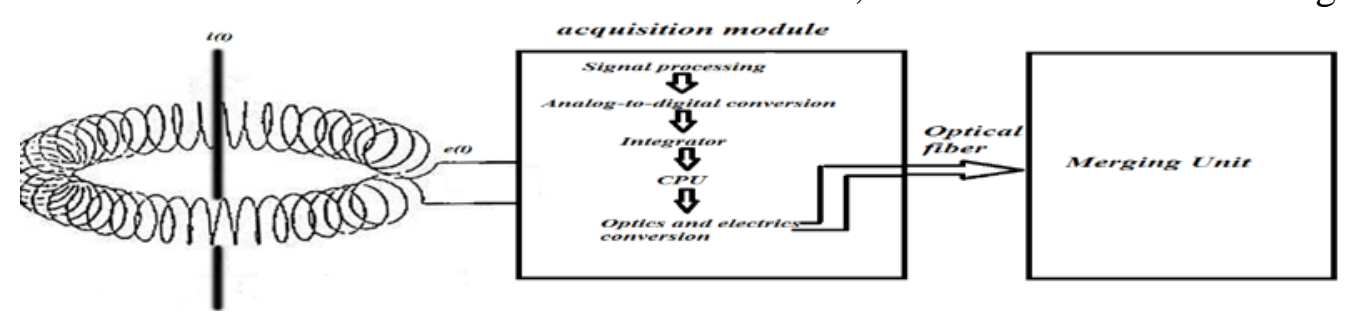

Figure1. The structure of current transformer based on Rogowski coil

When the current through the rogowski coil, based on electromagnetic induction principle, the coil is induced an electromotive force. So the equation of e(t) is in the form

$$
e(t)=-M \frac{d i(t)}{d t}
$$

Where $M$ is the mutual inductance,$i(t)$ is the primary current, $e(t)$ is the electromotive force .

The small signal by the coil induction will be processed of signal adjustment, integral transform, $\mathrm{AD}$ conversion and photoelectric conversion, the induction of small signal will be turned into the optical signal which is proportional to the primary current and conformed to the FT3 frame format .

Can be seen from the Figure 1, it may be the main factors influencing the transient performance of electronic current transformer with rogowski coil production installation process and the the selection of circuit parameters or integral algorithm in acquisition module. Then the transient performance will be analysed.

\section{The transient performance analysis}

The electronic current transformer has a good performance in the linearity, and because of the Sensors using non ferromagnetic materials, there is no magnetic saturation. So it has a good transfer performance for the aperiodic and harmonic component theoretically,but the output signal of rogowski coil that need to process by a series of electronic circuit can reduce the signal which is proportional to the primary current.

The aperiodic and harmonic compnent of transient processing may exert some influence on the magnitude and frequency response of the electronic circuit, then in the whole process of signal processing, the most important thing is the integral .In the practical application of integral circuit is mainly divided into analog and digital integral, then the transient characteristics of two different integration method is analyzed respectively.

\subsection{The transient performance analysis of analog integral}

At present, in the practical application, the analog integrator is realized by the integrated operational amplifier through the resistance $R$ and the feedback capacitance $C$ and the feedback resistance $R_{f}$, as shown in figure 2 .

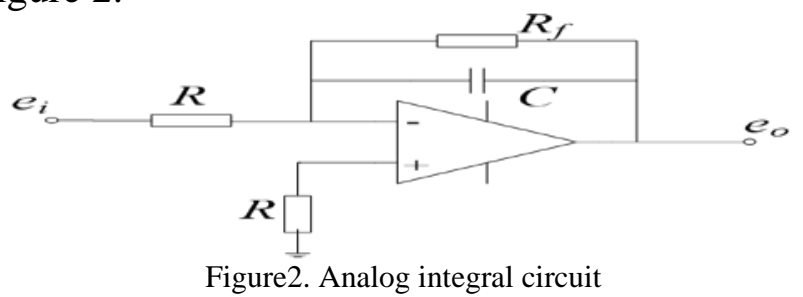


According to figure 2, the transfer function of analog integral can be shown as

$$
H(s)=-\frac{R_{f}}{R} \frac{1}{R_{f} C s+1}
$$

When the feedback capacitance parameters are selected with different values, the phasefrequency characteristics are shown in figure 3:

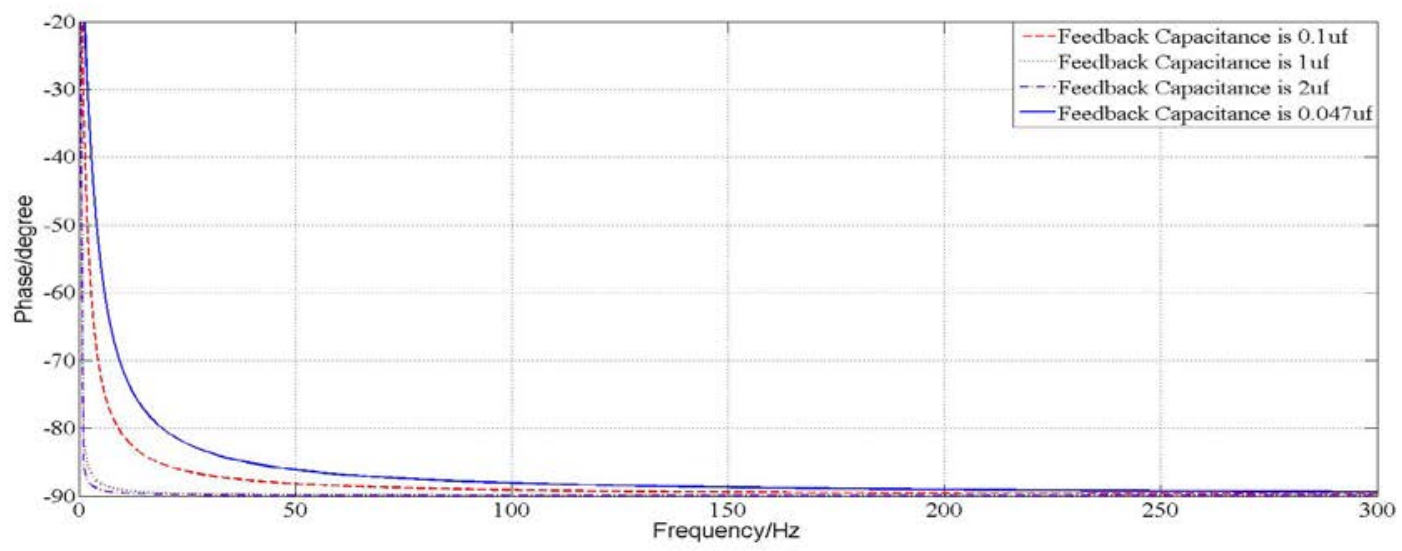

Figure 3. Phase-frequency characteristics with different values

The figure 3 shows that When the feedback capacitance is selecting the different parameters, the frequency response has a major difference in the low frequency, the smaller the value of feedback capacitance is, the larger the error is in the low frequency range (phase frequency characteristics should be offset 90 degree under ideal conditions). When the power system is at fault or disturbance, there may be a harmonic component and non periodic components in the input end of the integrator, when feedback capacitance parameters are selected inappropriately, the transient error will increase, the transient characteristics will be affected. According to the simulation results, it is shown that the transient characteristics can be effectively improved by increasing the feedback capacitance properly.

\subsection{The transient performance analysis of digital integral}

Digital integral is using specific software algorithms to restore the signal, the digital integral characteristics are mainly determined by the characteristics of software integral algorithm. There are three kinds of common integration algorithms:rectangular integral algorithm, trapezoidal integral algorithm and Simpson integral algorithm. In order to analyze the characteristics of the different frequencies, the transfer functions of the rectangular integral algorithm, the trapezoidal integral algorithm and the Simpson integral algorithm are deduced according to the principle of numerical integration ,the transfer functions are in the form

$$
\begin{gathered}
H(\omega)=\frac{T}{2} \frac{\left(e^{j \omega}+1\right)}{\left(e^{j \omega}-1\right)} \\
H(\omega)=\frac{T}{3} \frac{\left(e^{2 j \omega}+4 e^{j \omega}+1\right)}{\left(e^{2 j \omega}-1\right)}
\end{gathered}
$$

Where the $w$ is angular phase-frequency

$$
H(\omega)=\frac{T}{e^{j \omega}-1}
$$

frequency,the $T$ is sampling period.Then the characteristics of digital integral are shown in 
figure4:

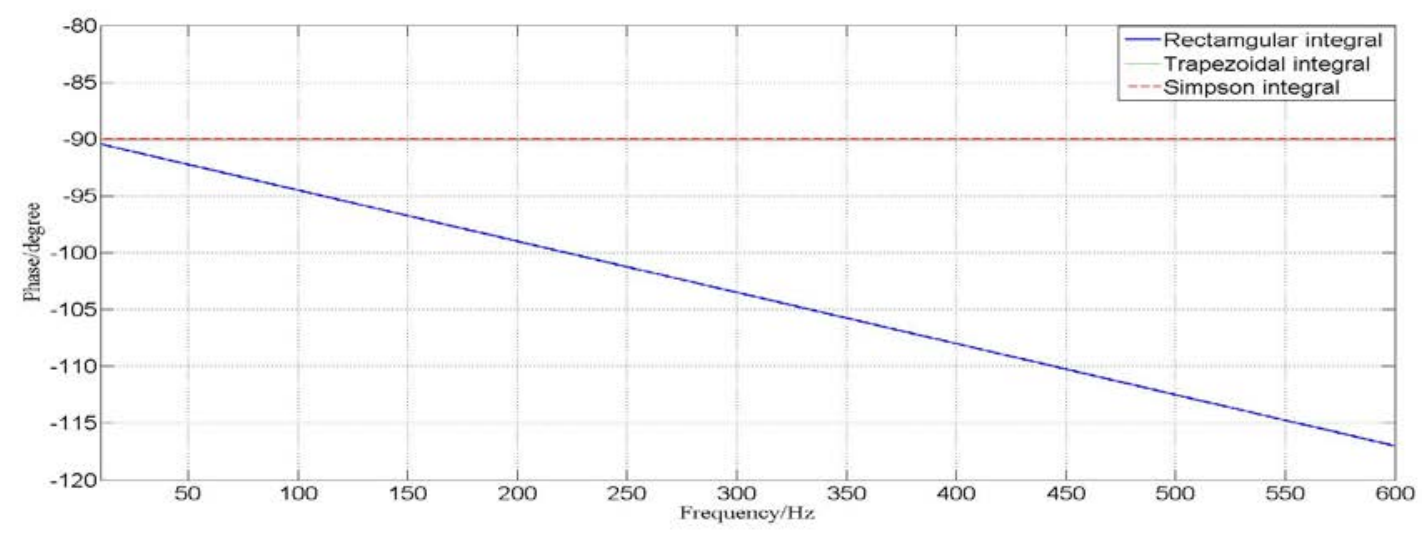

Figure 4. Phase-frequency characteristics with different integral algorithms

As can be seen from figure 4, the difference of the phase frequency characteristics have a major difference when selecting different integral algorithm, especially the phase error of rectangular integral algorithm increase with increasing frequency in the high frequency component, it has certain influence on transient characteristics of electronic current transformer because. When the trapezoidal integral algorithm and the Simpson integration algorithm are selected, the characteristics of the algorithm are not affected by the frequency and have a good transient response characteristics.

\section{Studying on transient performance testing}

From the above, it is shown that the selection of different integral form and circuit device parameters and different software integration algorithms have a great influence on the integral effect, especially it has a major influence for some specific harmonic components. In addition, the electronic current transformer operating environment is bad, its transient characteristics will be affected because of the electromagnetic interference, environmental temperature.

Therefore, it is necessary to test for the transient characteristics of the electronic current transformer.The traditional testing method is using transient dynamic simulation testing system, the method has the advantages that the whole transient process can be truly reflected and the phenomenon is obvious, the disadvantage is that it is difficult to realize the large transient current.

Aiming at the limitations of traditional testing methods, this paper puts forward a kind of electronic current transformer transient testing method based on RTDS simulation, the method mainly uses the RTDS simulation system to replace the traditional dynamic simulation system, it realizes the connection of power system simulation and actual electronic current transformer, establishes the real-time running state. To carry out the relevant characteristic test based on digital real-time simulation technology, such as steady state and transient characteristics.

\subsection{Testing platform based on RTDS simulation}

RTDS is a special device used to study the electromagnetic transient phenomena of power system, which is composed of hardware system and software system . At present, RTDS has a wide range of applications, such as: electromagnetic transient simulation, fault repeat, secondary equipment closed loop testing, high voltage DC control and protection testing and smart substation equipment testing( ZHOU Bowen\&YANG Jun,2013 and XU Zhi-heng\& QIAN Luo-jiang,2009).

The transient testing method of electronic transformer based on the RTDS simulation is mainly used in the laboratory testing. The idea is to adopt the closed loop simulation program of the RTDS simulation system, which is based on the ratio of the field installed electronic transformer, The 
power system model is established based on RTDS, it can adjust the output power and load, set a fault in any place, and make the voltage and current under the real state of normal operation or the accident situation through the analog power amplifier to convert the analog signal for electronic transformer.Then, the signal is transmitted to the merging unit in the FT3 frame format, and the merging unit is converted into a IEC61850-9-2LE network packet. RTDS is used to simulate the transient process in the input of the electronic transformer, and the transient response of the electronic transformer is qualitatively analyzed by comparing the transient response of the standard source by the network packet analyzer . The testing platform is shown in figure 5:

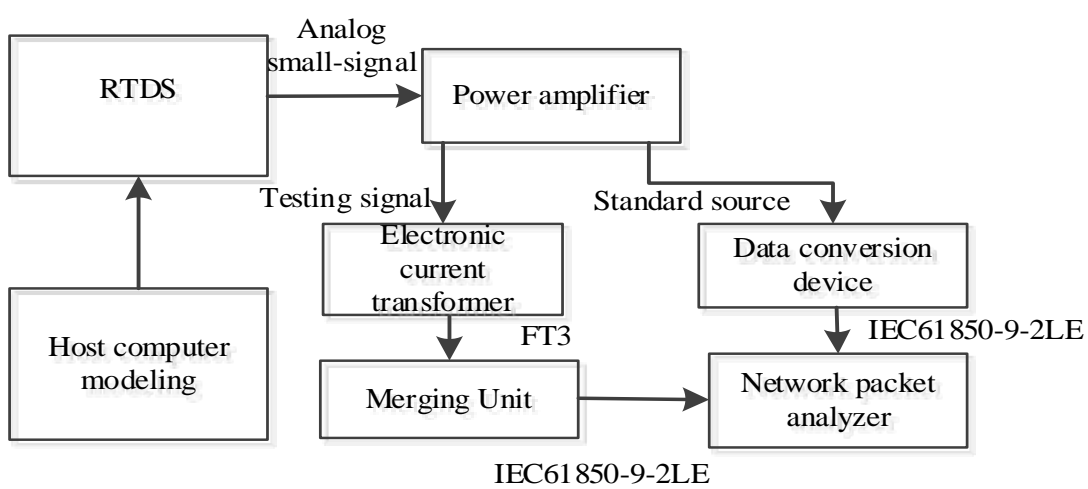

Figure 5. Testing platform based on RTDS

To validate the above testing method, this paper build a testing platform based on RTDS simulation, as shown in Figure 5,It builds a simple model for a single machine infinite real-time operating environment based on RTDS and simulates the dynamic process,then the voltage and current calculation data by the RTDS's GTAO board is converted to analog small-signal, and then the analog small-signal is converted to the current analog that is applicable for electronic transformer by using power amplifier, one current signal as measured source input to the electronic transformer and merging unit, another current signal as a standard source (the data conversion device to complete the analog signal into the message data format based on IEC61850-9-2LE), the two signals simultaneously access the network packet analysis device, The transient response characteristics of electronic transformer are analyzed qualitatively by comparing the measured source and standard source.

The following aspects is considered mainly in the laboratory of electronic transformer for carrying out the relevant tests based on RTDS simulation: 1) response of electronic transformer in fault transient process 2) response to the high frequency signal 3) response to the power supply power-down and recovery 4) response in the condition that power supply is not stable.The part of the test results are shown in figure 6 7.

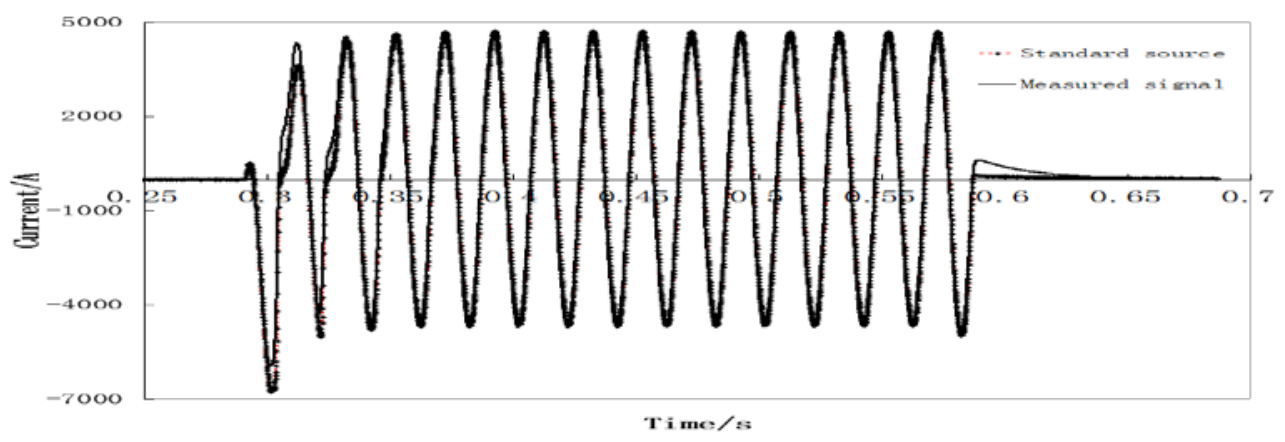

Figure 6. Response of electronic transformer in fault transient process 


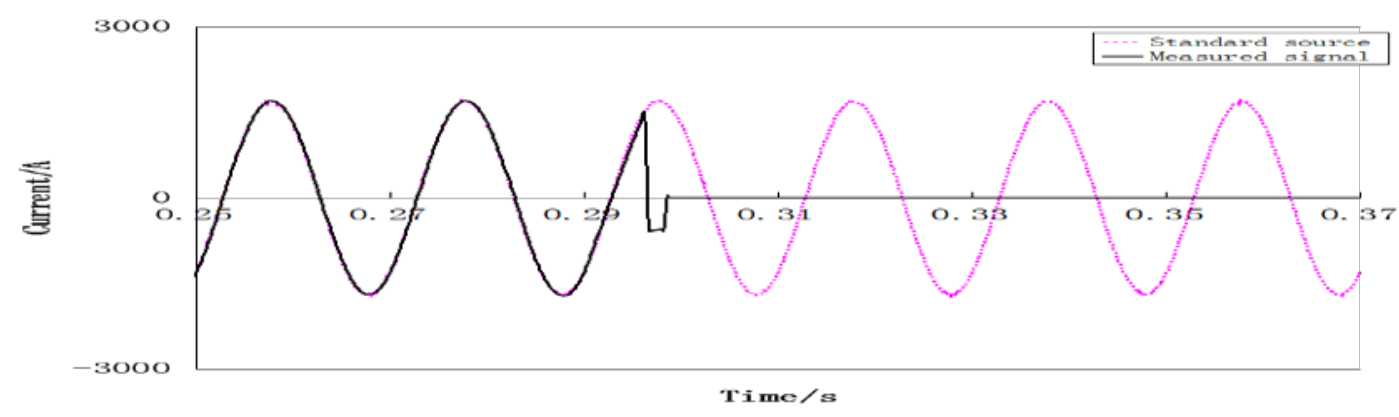

Figure 7. Response of electronic transformer under the power-down condition

The test results show that there are problems in the transient response of electronic transformer at the laboratory,such as the response of electronic transformer and standard source is not the same under the influence of aperiodic and harmonic component when the fault occurs. In addition, there is a delay in the electronic transformer when the fault disappears. And the electronic transformers can't respond to high frequency signal correctly. Besides the electronic transformers will have a shock current when the power drops. The test results also verify the theoretical analysis, the transient performence may be influenced by the structure and parameters of the circuit, and the necessity of transient testing is proved. The electronic transformer transient testing method based on RTDS simulation can reflect the transient process of the response intuitively, the test results further verify the validity and feasibility of the method.

\section{Conclusion}

Theory analysis shows that the transient characteristics of electronic current transformer have a certain influence when the selection of integral circuit parameters and integral algorithm are not appropriate. In order to evaluate the performance of electronic transformer, the transient characteristics of electronic current transformer are tested except for its steady-state error testing. This paper proposes the method that combined RTDS simulation with the actual electronic transformer based on studying on the transient performance test method. The dynamic response of the electronic transformer to the fault process and high frequency and the power supply failure is checked.The test system can evaluate the performance of the electronic transformer and provide reliable data support for the practical operation.

\section{References}

[1] ZHAO Yong,KONG Shengli,LUO Qiang. Research on testing technology of ECT transmission delay time in transient state[J].Power System Protection and Control,2014,42(17):125-129.

[2] LI Wei, YIN Xianggen, CHEN Deshu, Transient characteristics of Rogowski coil-based current sensor[J], Electric Power Automation Equipment,2008, 28(10):34-37.

[3] ZHANG Kewei,WANG Ning,DUAN Xiongying.A Digital Integrator For Electronic Current Transducer[J].Proceedings of the CSEE,2004,24(12):104-107.

[4] ZHOU Bowen, YANG Jun, SONG Xinli, et al. Interface simulation platform of stability control device in dynamic simulation of power system [J]. Electric Power Automation Equipment, 2013, 33(10):157-161.

[5] XU Zhi-heng, QIAN Luo-jiang. Application method of RTDS user define component model (UDC) [J]. Power System Protection and Control, 2009, 37(22):141-145. 